\title{
Electrochemical Reduction of the Simplest Monosaccharides: Dihydroxyacetone and Glyceraldehyde
}

\author{
Zhiqin Liang, Matias A. Villalba, Giulia Marcandalli, Kasinath Ojha, Arthur J. Shih, \\ and Marc T. M. Koper*
}

Cite This: ACS Catal. 2020, 10, 13895-13903

Read Online

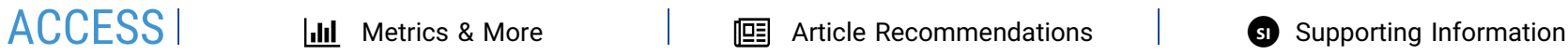

ABSTRACT: Dihydroxyacetone (DHA) and glyceraldehyde (GA) - the simplest monosaccharides in nature-are both essential building blocks in organic synthesis. There have been detailed investigations on the selective production of DHA and GA through the platinum-catalyzed electrochemical oxidation of glycerol, which is a surplus byproduct from biodiesel. However, less attention has been paid to the electrochemical behavior of DHA and GA themselves. Here, we report the electrochemical reduction of DHA and GA at different $\mathrm{pH}$ on platinum and palladium electrodes. Palladium exhibits a superior activity toward both the dehydroxylation and hydrogenation of DHA and GA. Using online high-performance liquid chromatography, we show that both DHA and GA display a two-step reduction. In particular, DHA is reduced first to acetol and then sequentially to acetone, while GA is first reduced to 3-hydroxypropionaldehyde (3-HPA), followed by hydro-

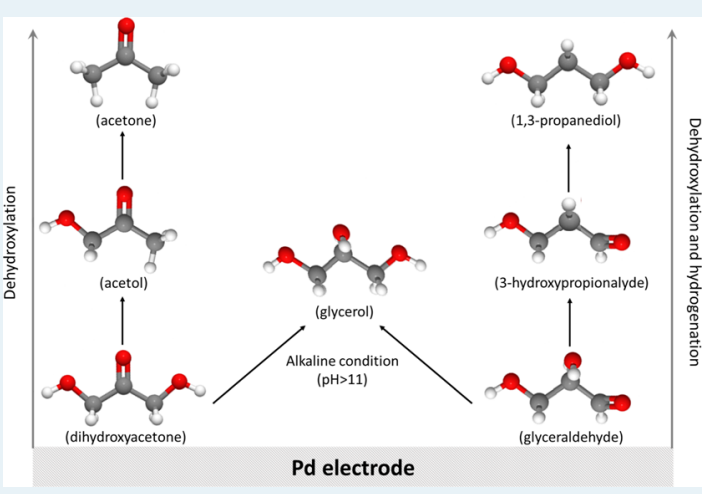
genation to 1,3-propanediol. The reduction of both molecules is accompanied by a poisoning of the catalyst surface. In situ Fourier-transform infrared spectroscopy measurements suggest adsorbed carbon monoxide as the poisoning species, resulting from the dissociative adsorption of DHA and GA. Mechanistic reaction pathways for both DHA and GA reduction are proposed and discussed.

KEYWORDS: dihydroxyacetone, glyceraldehyde, electrochemical reduction, organic synthesis

\section{INTRODUCTION}

Gasoline and diesel still constitute the largest supply for the increasing global fuel demand. Addressing the increasing concerns regarding the sustainability of using fossil sources stimulates research into the conversion of renewable feedstock to value-added chemicals and fuels.

Glycerol, a surplus byproduct from the large production of (renewable) biodiesel, can be converted through hydrogenolysis, fermentation, dehydration, carboxylation, and esterification $^{1,2}$ into a number of industrially desired chemicals, such as 1,3-propanediol (1,3-PD) and 1,2-PD. However, these routes either require pyrolysis and hydrogen supply or depend exclusively on biological metabolism.

Electrochemical routes provide an alternative approach toward glycerol conversion. In previous work, we reported the selective electrochemical oxidation of glycerol to dihydroxyacetone (DHA) and glyceraldehyde (GA)..$^{3-5}$ Both DHA and GA could be further oxidized to their corresponding acids, such as hydroxypyruvic acid, glyceric acid, and glycolic acid.

On the other hand, the selective reduction of glycerol is particularly challenging due to the negative reduction potential of their hydroxyl groups. ${ }^{6}$ A recent work proposed to bypass this problem by combining the non-electrochemical dehy- dration of glycerol to acetol with the subsequent electrocatalytic reduction of acetol to valuable commodities. ${ }^{7}$ Alternatively, direct electrochemical conversion of glycerol can be conducted, wherein glycerol is first oxidized selectively, followed by the reduction of the produced intermediates. ${ }^{8,9}$ The selectivity of the desired product is relatively low in this process because of the broad product distribution, which can be ascribed to the undivided cathode and anode reactions. Very recently, one-step electroreduction of glycerol was achieved to produce 1,2-PD and other byproducts, ${ }^{10}$ during which an acid catalyst (Amberlyst-15), high temperature, and large overpotential were required. The reaction mechanism is still unclear.

The discussion above indicates that there is still room to explore simple and clean avenues to upgrade the biomass feedstock. In previous work, we have shown that glycerol can be electrochemically oxidized to GA and DHA with high

Received: September 21, 2020

Revised: October 28, 2020

Published: November 16, 2020 
Scheme 1. Possible Reaction Routes for the Synthesis of Valuable Commodities from Glycerol Biomass Feedstock

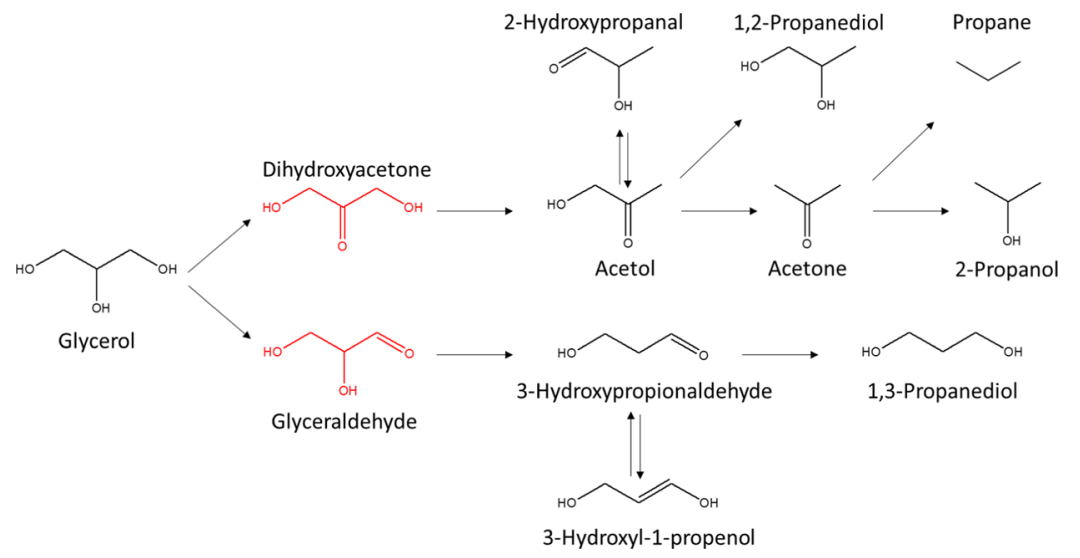

selectivity using platinum and modified platinum electrodes, respectively. ${ }^{3-5}$ Both products are the simplest monosaccharides, which could be potentially hydrogenated or dehydroxylated, as presented in Scheme 1. Combining selective electrooxidation of glycerol at the anode and subsequent selective electro-reduction of the products at the cathode could provide a way forward in generating valuable products from glycerol using electrochemistry.

Early work suggested that DHA and GA can indeed be reduced electrochemically and showed polarographic reduction waves on the mercury electrode that were dependent on different $\mathrm{pH}$ conditions. ${ }^{1,12}$ However, the polarographic signals do not give information about the products formed. In view of this, the present work is devoted to a study on the DHA and GA electroreduction in a wide range of $\mathrm{pH}$ using catalytic electrodes, that is, platinum and palladium. Combined with the results of online high-performance liquid chromatography (HPLC) and in situ Fourier-transform infrared spectroscopy (FTIR), we obtain detailed insight into the reaction pathways of dehydroxylation and hydrogenation of DHA and GA.

\section{EXPERIMENTAL SECTION}

Chemicals and Electrolysis. Linear sweep voltammetry (LSV) experiments were performed in a one-compartment three-electrode single cell at room temperature and ambient pressure. Bulk electrolysis experiments were performed in an $\mathrm{H}$-type cell divided by a proton exchange membrane. The used glassware was stored in an acidic permanganate solution. Prior to use, the glassware was rinsed with the acidic peroxide solution and MilliQ water, followed by boiling several times with MilliQ water.

The chemicals used for the electrolyte preparation were high-purity perchloric acid (Merck Suprapur, 70\%), sodium perchlorate (Sigma-Aldrich, 99.99\%), phosphoric acid (Merck Suprapur, $85 \%$ ), sodium phosphate mono- and dibasic (SigmaAldrich, >99\%), sodium phosphate tribasic (Sigma-Aldrich, $>98 \%$ ). DHA (99\%), DL-GA (>90\%), acetol (>95\%), acetone (99.9\%), and 1,3-PD (98\%) were purchased from SigmaAldrich. 3-Hydroxypropionaldehyde (95\%) was purchased from MolPort. All chemicals were used without further purification. A platinum wire and a home-made reversible hydrogen electrode (RHE) were used as the counter and reference electrodes, respectively. Before each experiment, the electrolyte was deaerated with argon (Linde, 6.0 Scientific) for $30 \mathrm{~min}$.
Electrochemical measurements were performed on a BioLogic VSP300 potentionstat with iR-correction during the LSV scan or the bulk electrolysis. Experiments were carried out in unbuffered perchlorate solution and in phosphate buffer solution in the $\mathrm{pH}$ range between 5 and 11 (Table $\mathrm{S} 1$ in the Supporting Information). Before the experiment, the platinum $(\mathrm{Pt})$ and palladium $(\mathrm{Pd})$ working electrodes with a diameter of $1 \mathrm{~cm}$ were polished using diamond $(1,0.25$, and $0.05 \mu \mathrm{m})$. Next, the electrode was sonicated in MilliQ water for 5 min. The electrode was then dipped into a fresh $0.1 \mathrm{M} \mathrm{H}_{2} \mathrm{SO}_{4}$ solution to run cyclic voltammetry (CV) from 0.05 to $1.55 \mathrm{~V}$ until a stable standard CV curve was observed.

The surface morphology of the Pd electrode was measured using a Thermo Scientific Apreo S scanning electron microscope with a $15.00 \mathrm{kV}$ and $0.10 \mathrm{nA}$ electron beam using an Everhart-Thornley detector. The surface morphology was observed to be amorphous (Figure S1a,b).

The electrochemically active surface area of $\mathrm{Pd}$ was measured using $\mathrm{CV}$ by determining the charge of a full monolayer of PdO (Figure S1c,d)..$^{13,14}$ The current densities in the main text are normalized by the electrochemically active surface area.

Online HPLC Measurement. HPLC was employed to analyze the non-volatile products. ${ }^{15} 50 \mathrm{mM} \mathrm{DHA}$ or GA was dissolved in $100 \mathrm{~mL}$ of the electrolyte in a one-compartment cell. The products were collected during the voltammetric scan with a small Teflon tip (with an inner diameter of $0.38 \mathrm{~mm}$ ) positioned $c a .10 \mu \mathrm{m}$ from the center of the electrode surface. The other side of the tip was connected to a PEEK capillary with inner/outer diameters of $0.13 / 1.59 \mathrm{~mm}$. Owing to the local sampling and the low product concentration, there is no significant effect on the oxidation of products at the counter electrode.

Samples with a volume of $60 \mu \mathrm{L}$ each were collected with a fraction collector (FRC-10A, Shimadzu) at the flow rate of 60 $\mu \mathrm{L} / \mathrm{min}$ (LC-20AT pump, Shimadzu). The scan rate of the potential sweep during sample collection was set to $1 \mathrm{mV} \mathrm{s}^{-1}$ scanning from $0 \mathrm{~V}$ to $-2.0 \mathrm{~V}$ versus RHE. Therefore, each sample held the average concentration of a $60 \mathrm{mV}$ potential interval. The $\mathrm{pH}$ of the samples after carrying out the voltammetric scan with online HPLC was measured by mixing all of the collected samples together.

The samples were analyzed with HPLC (Prominence HPLC, Shimadzu). The samples were placed in an autosampler (SIL-20A) which injected $20 \mu \mathrm{L}$ of the sample into the column. An Aminex HPX 87-H (Bio-Rad) column together 
a

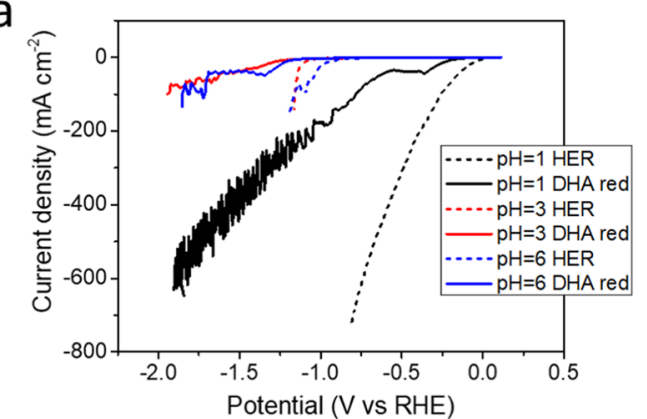

b

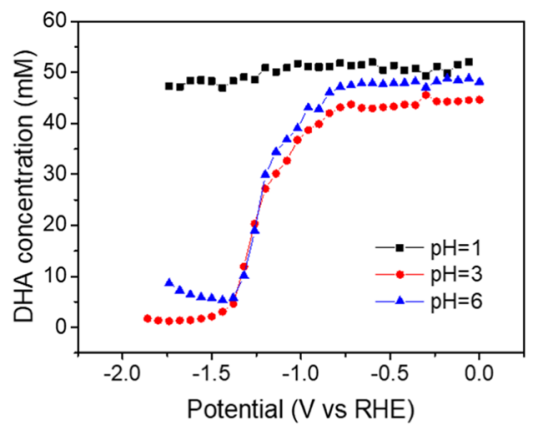

e

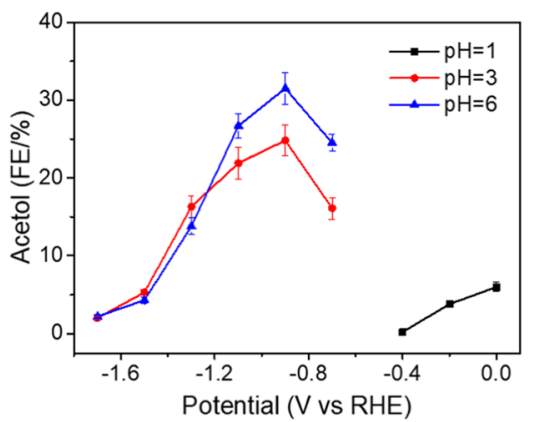

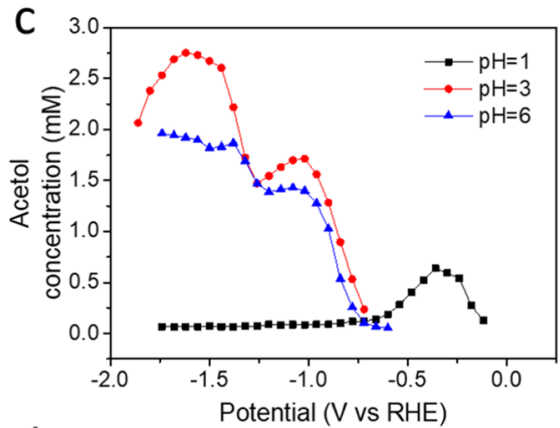

d

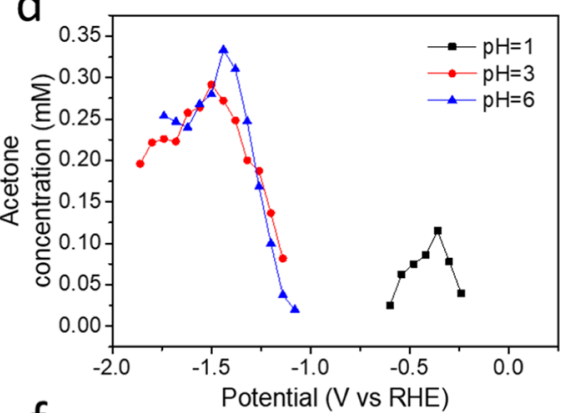

$\mathrm{f}$

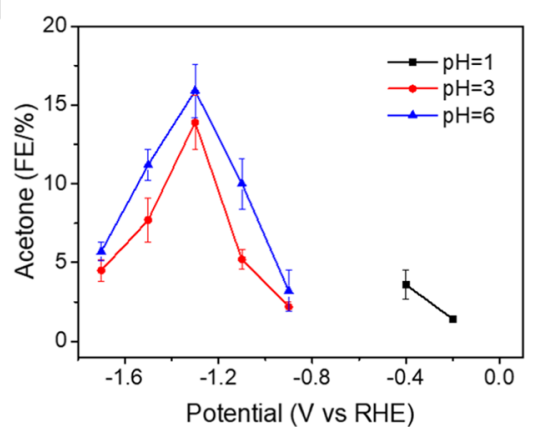

Figure 1. $\mathrm{pH}$-dependence of DHA $(50 \mathrm{mM})$ reduction on Pd electrode and the corresponding product identification. (a) LSV in three unbuffered perchlorate electrolytes recorded concomitantly with the sample collection. The scan rate is $1.0 \mathrm{mV} \mathrm{s}^{-1} \cdot \mathrm{pH}=1: 0.1 \mathrm{M} \mathrm{HClO}$; $\mathrm{pH}=3: 0.001 \mathrm{M}$ $\mathrm{HClO}_{4}+0.099 \mathrm{M} \mathrm{NaClO}_{4}$; and $\mathrm{pH}=6: 0.1 \mathrm{M} \mathrm{NaClO}_{4}$. The dashed line indicates the scan without DHA, while the solid line is with DHA. (b-d) Corresponding concentrations of (b) DHA reactant, (c) first product acetol, and (d) second product acetone as a function of the applied potential. $(\mathrm{e}, \mathrm{f}) \mathrm{FE}$ of the products (e) acetol and (f) acetone at different potentials from -0.7 to $-1.7 \mathrm{~V} v \mathrm{RHE}$.

with a Micro-Guard Cation H Cartridge (Bio-Rad) in front of the column was used. The eluent was $5 \mathrm{mM} \mathrm{H}_{2} \mathrm{SO}_{4}$, and the

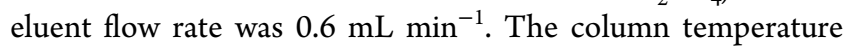
was set to $45{ }^{\circ} \mathrm{C}$ in a column oven (CTO-20A), and the separated compounds were detected with a refractive index detector (RID-10A).

Bulk electrolysis for determining Faradaic efficiencies (FEs) of DHA and GA reductions was performed in the H-cell divided by a Nafion proton exchange membrane. Each compartment contained $10 \mathrm{~mL}$ of the electrolyte with 50 $\mathrm{mM}$ molecule solution. Each reaction lasted for $60 \mathrm{~min}$ with a manual collection of $20 \mu \mathrm{L}$ samples and analyzed by HPLC. The data reported are the average of two independent experiments with fresh electrodes. The FE for the reduction product is calculated using the following equation

$$
\mathrm{FE}=\frac{m \times n \times F}{Q}
$$

where $m$ is the mol of the reduction product, $n$ is the number of electrons needed for the corresponding product, $F$ is the Faraday constant, and $Q$ is the total charge measured during the reaction.
In Situ FTIR Measurement. In situ FTIR measurements were performed to detect possible adsorbed species during DHA/GA reduction. The measurements were carried out with a Bruker VERTEX $80 \mathrm{~V}$ IR spectrophotometer. $\mathrm{A} \mathrm{CaF}_{2}$ prism beveled at $60^{\circ}$ was used, and the spectra correspond to an average of 300 interferograms with $8 \mathrm{~cm}^{-1}$ resolution. The experiments were performed in the thin-layer configuration, where the working electrode was pressed onto the prismatic window and the solution species got trapped, meaning there was only slow diffusion in or out of the thin layer.

The spectra were obtained in the wavenumber range between 2750 and $1100 \mathrm{~cm}^{-1}$. All spectra in this work are presented as transmittance, according to $T=R / R_{0}$, where $R$ and $R_{0}$ are the reflectance corresponding to the single-beam spectra obtained at the sample and reference potentials, respectively. Therefore, positive bands correspond to the species consumed, and negative bands to the species formed with increasingly negative potential.

\section{RESULTS AND DISCUSSION}

CV and Online HPLC. Since Pt is a good catalyst for the selective oxidation of glycerol, in the initial experiments we reduced DHA and GA on the Pt electrode. However, only a 

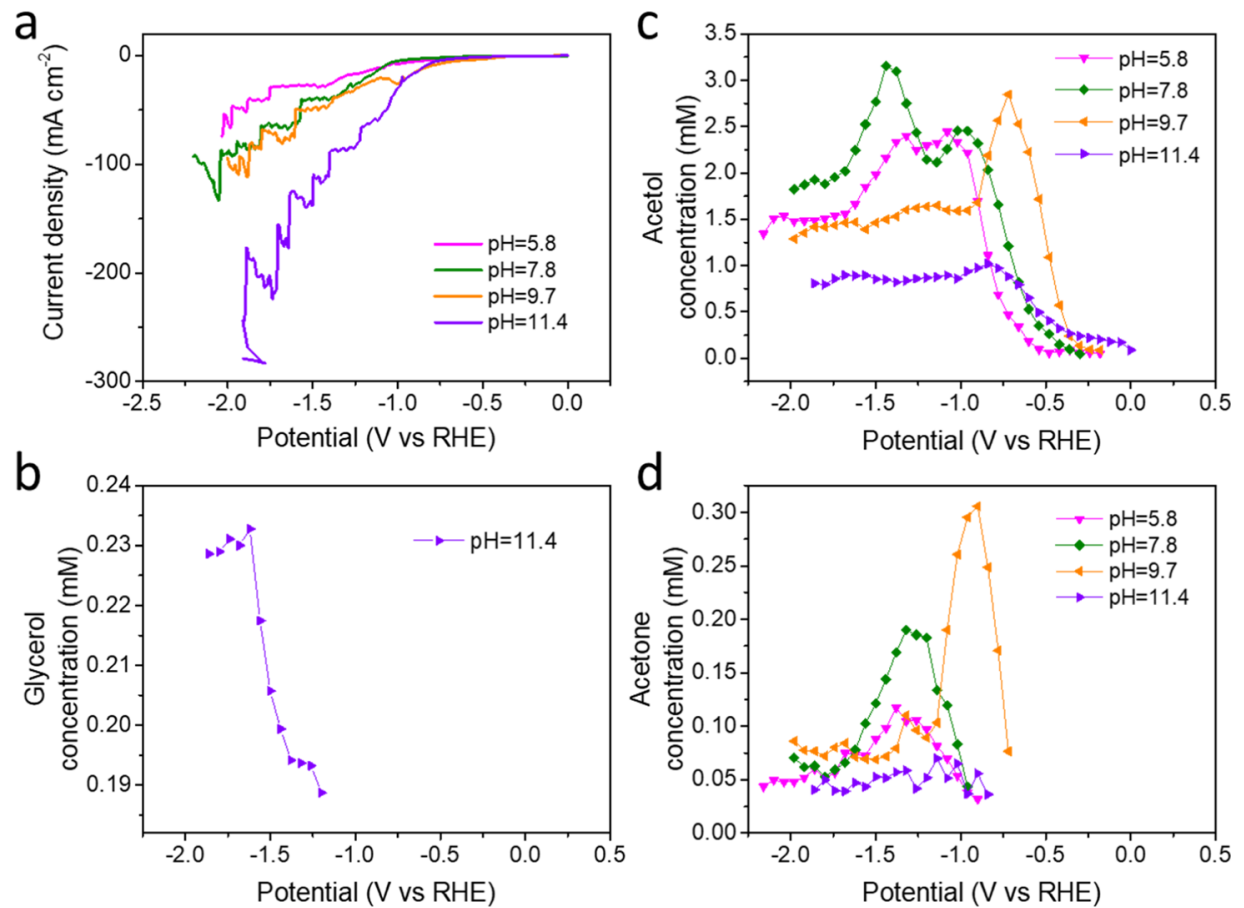

Figure 2. DHA reduction in phosphate buffer electrolyte. (a) LSV at different $\mathrm{pH}$. The scan rate is $1.0 \mathrm{mV} \mathrm{s}^{-1}$. (b-d) Corresponding product concentrations of (b) glycerol, (c) acetol, and (d) acetone as a function of the applied potential.

negligible amount of acetol was observed (Figure S2). We then carried out the reaction on a Pd electrode, which is a popular metal catalyst in the hydrogenation of imines, olefins, ketones, and other organic compounds. ${ }^{16}$ Comparing the results on both electrodes, we found that significant concentrations of acetol and acetone were produced using Pd catalyst (Figure S2b), while Pd showed a lower total current density compared to Pt (Figure S2a), indicating a higher product selectivity on the Pd electrode. Therefore, for the rest of the paper, we consider only the results obtained on the Pd electrode.

Figure 1 shows the LSV at $1 \mathrm{mV} \mathrm{s}^{-1}$ in unbuffered $0.1 \mathrm{M}$ perchlorate solution with $50 \mathrm{mM}$ of DHA at bulk $\mathrm{pH}$ of 1,3 , and 6 , together with the corresponding products detected by online HPLC. We note that the voltammetry in Figure 1 and in subsequent figures looks noisy and distorted because of the disturbance by the tip-based online fraction collection ${ }^{15}$ and hydrogen bubbles at negative potentials.

For all three $\mathrm{pH}$ values, the reduction currents are significantly suppressed after adding DHA (Figure 1a), which we interpret as a blockage of hydrogen evolution. This observation indicates that the presence of DHA inhibits hydrogen evolution probably due to the adsorption of a poisoning species on the electrode surface. We will come back to the nature of this species when we discuss the in situ FTIR spectroscopy measurements (see below). A similar poisoning phenomenon has also been observed during glucose hydrogenation. $^{17}$

Figure $1 \mathrm{~b}$ shows that DHA concentration decreases only slightly with more negative applied potential at $\mathrm{pH}=1$, while at $\mathrm{pH}=3$ and 6, DHA decreases substantially when the potential is more negative than $-0.75 \mathrm{~V}$ versus RHE. In addition to direct DHA reduction, this could be associated with side reactions, such as isomerization between DHA and $\mathrm{GA}^{18}$ and aldol condensation of both trioses to hexoses ${ }^{19}$ (Figure S3), as a result of the alkaline local pH caused by the increased hydrogen evolution and dehydroxylation reactions
(Table S2). At $\mathrm{pH}=1$, high proton concentration attenuates the rise in local $\mathrm{pH}$, thereby suppressing the possible side reactions.

Figure 1c,d displays the product distribution of DHA reduction for the three $\mathrm{pH}$ values. At $\mathrm{pH}=1$, the first product acetol is formed at an onset potential of $-0.12 \mathrm{~V}$ versus $\mathrm{RHE}$, with the concentration increasing until $-0.36 \mathrm{~V}$ versus $\mathrm{RHE}$, after which it decreases. With a small delay, acetone is formed as well (Figure $1 \mathrm{~d}$ ). At $\mathrm{pH}=3$ and 6 , acetol is produced at a considerably more negative potential, $c a$. $-0.66 \mathrm{~V}$ versus $\mathrm{RHE}$, with an increased concentration observed until $-1.02 \mathrm{~V}$ versus RHE. Acetone formation starts from ca. $-1.15 \mathrm{~V}$ versus RHE for both $\mathrm{pH}=3$ and 6 , indicating that acetol is a likely intermediate in the conversion of DHA to acetone. This is confirmed by a separate experiment in which acetol is reduced, indeed leading to the production of acetone (Figure S4a,b), at the same potential as observed in Figure 1d.

Since $\mathrm{pH}$ was increased during the sample collection (Table S2), we compared the results of the samples that were quenched or not. The quenched samples were prepared by adding $60 \mu \mathrm{L}$ of $0.1 \mathrm{M} \mathrm{HClO}_{4}$ to each collected sample. Online HPLC results show that quenching the samples does not have a major effect on the onset potential or the product distribution (Figure S5).

From the above observations, we conclude that in acid and neutral electrolytes, DHA-with two terminal $\mathrm{OH}$ groups and one ketone group-prefers to break the $\mathrm{C}-\mathrm{OH}$ group adjacent to the $\mathrm{C}=\mathrm{O}$ group rather than the direct attack of the carbonyl group. This has also been observed in the electrochemical deoxygenation of glucose ${ }^{17}$ and other oxygenates. ${ }^{20}$ We did not observe a significant production of glycerol at the employed $\mathrm{pH}$ conditions, which might be a result of low concentration that is below the detection limit.

For a quantitative analysis of the reduction products, we performed one-hour bulk electrolysis for DHA reduction at three different $\mathrm{pH}$ (Figure S6 and Table S4). The results show 
a

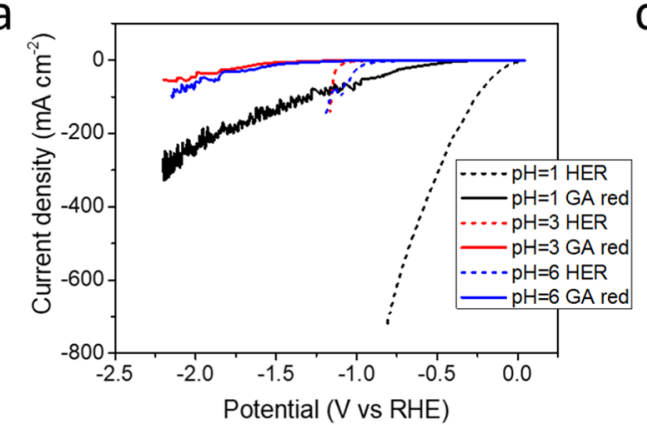

b

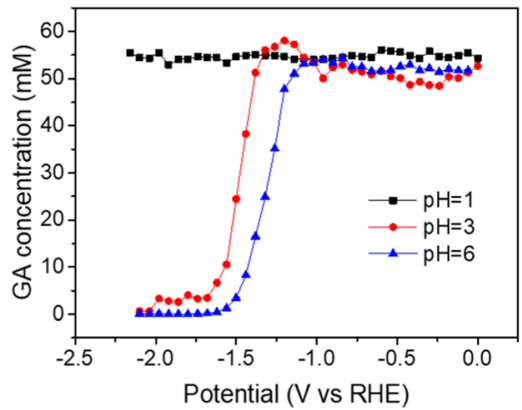

e

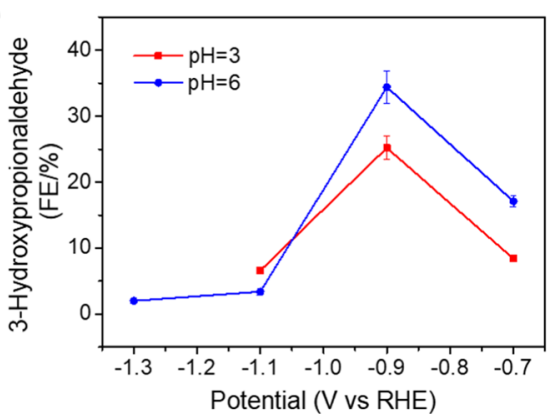

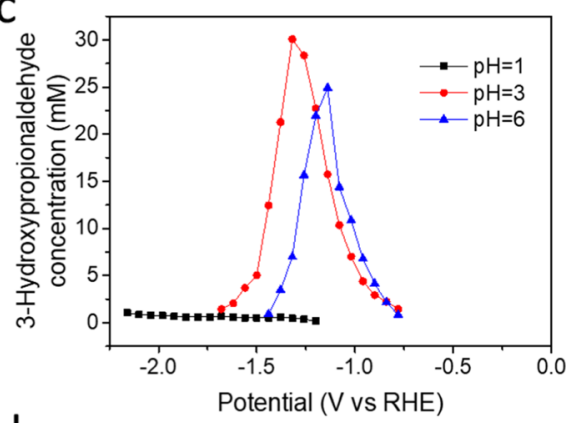

d

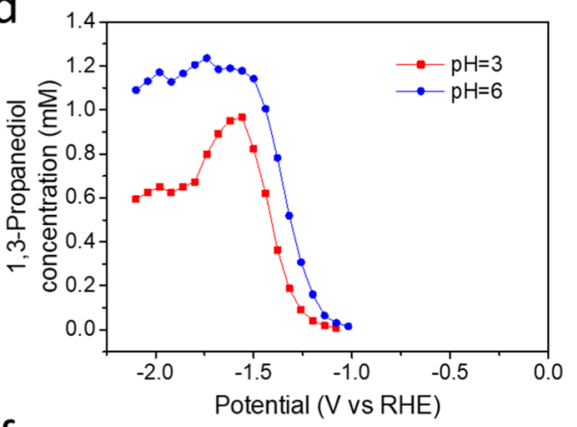

$f$

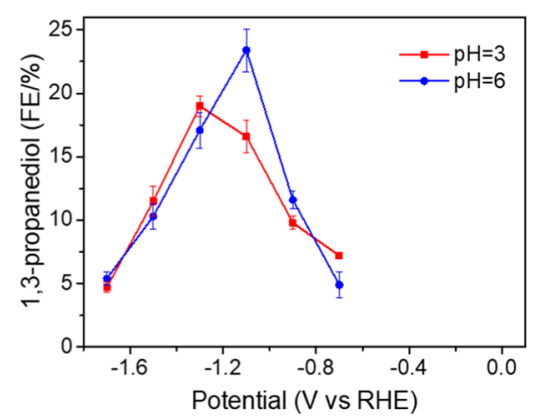

Figure 3. $\mathrm{pH}$-dependence of $\mathrm{GA}(50 \mathrm{mM})$ reduction on $\mathrm{Pd}$ electrode and the corresponding product identification. (a) LSV in the three unbuffered perchlorate electrolytes. The scan rate is $1.0 \mathrm{mV} \mathrm{s}^{-1}$. $\mathrm{pH}=1: 0.1 \mathrm{M} \mathrm{HClO}_{4} ; \mathrm{pH}=3: 0.001 \mathrm{M} \mathrm{HClO}_{4}+0.099 \mathrm{M} \mathrm{NaClO}_{4}$; and $\mathrm{pH}=6$ : $0.1 \mathrm{M} \mathrm{NaClO}_{4}$. The dashed line indicates the scan without GA, while the solid line is with GA. (b-d) Corresponding concentrations of (b) GA reactant, (c) first product 3-HPA, and (d) second product 1,3-PD as a function of the applied potential. (e-f) FE of the products (e) 3-HPA and (f) $1,3-\mathrm{PD}$ at different potentials from -0.7 to $-1.7 \mathrm{~V}$ vs RHE.

that under these conditions, acetol has a maximum $\mathrm{FE}$ of $31 \%$ at $-0.9 \mathrm{~V}$ versus $\mathrm{RHE}$ for $\mathrm{pH}=6$ (Figure $1 \mathrm{e}$ ). At the same $\mathrm{pH}$, acetone shows the highest efficiency of $16 \%$ at $-1.3 \mathrm{~V}$ versus RHE (Figure 1f).

Different phosphate buffer solutions with $\mathrm{pH}$ range from 5 to 11 were employed as well to investigate the $\mathrm{pH}$ effect on DHA reduction (Figure 2). We found that the product distribution was similar to that in the unbuffered solution. In addition, acetol concentration profiles are slightly shifted toward positive potentials with increasing $\mathrm{pH}$ on the $\mathrm{RHE}$ scale (Figure 2c). Acetone presents a similar trend except for $\mathrm{pH}=11.4$ since there is little acetone production at this $\mathrm{pH}$ condition (Figure 2d). At alkaline $\mathrm{pH}$, glycerol was also produced through the direct hydrogenation of the carbonyl group as an additional pathway (Figure $2 \mathrm{~b}$ ).

Figure 3 shows the results for GA reduction in unbuffered electrolytes at different $\mathrm{pH}$. Similar to DHA reduction, the presence of GA inhibits hydrogen evolution significantly (Figure 3a). Figure $3 \mathrm{~b}$ shows that GA concentration follows the same trend as DHA reduction at different $\mathrm{pH}$ : hardly any decrease at $\mathrm{pH}=1$ and a substantial decrease at $\mathrm{pH}=3$ and 6 . At $\mathrm{pH}=3$ and 6 , the increased hydrogen evolution and dehydroxylation/hydrogenation reactions at more negative potentials lead to a high local alkaline $\mathrm{pH}$, which gives rise to more isomerization and aldol condensation side reactions (Figure S7 and Table S3).

In contrast to $\mathrm{DHA}$ reduction, we find that at $\mathrm{pH}=1, \mathrm{Pd}$ is inactive for GA reduction. Only a negligible concentration of 3-HPA is observed at the relatively negative potential of -1.2 $\mathrm{V}$ versus RHE, while there is no further reduction of 3-HPA (Figure 3c). At $\mathrm{pH}=3$ and 6, 3-HPA is formed from a potential of $c a$. $-0.78 \mathrm{~V}$ versus RHE. The second product, 1,3$\mathrm{PD}$, starts to appear at $-1.08 \mathrm{~V}$ versus $\mathrm{RHE}$, with its concentration reaching a plateau when the concentration of 3HPA is decreasing and ultimately exhausted (Figure 3d), indicating that 3-HPA is the intermediate for the production of 1,3-PD. This is confirmed by a separate experiment in which 3HPA is reduced to 1,3-PD (Figure S4c,d) at the same potential as observed in Figure $3 \mathrm{~d}$.

We also conducted bulk electrolysis for GA reduction (Figure S8 and Table S5). The results show that 3-HPA and 1,3-PD are only detected at $\mathrm{pH}=3$ and $\mathrm{pH}=6$. At $\mathrm{pH}=6,3$ HPA achieves the highest selectivity of $35 \%$ at $-0.9 \mathrm{~V}$ versus RHE (Figure 3e). At the same $\mathrm{pH}$ condition, 1,3-PD has a maximum $\mathrm{FE}$ of $23 \%$ at $-1.1 \mathrm{~V}$ versus RHE (Figure $3 \mathrm{f}$ ). 

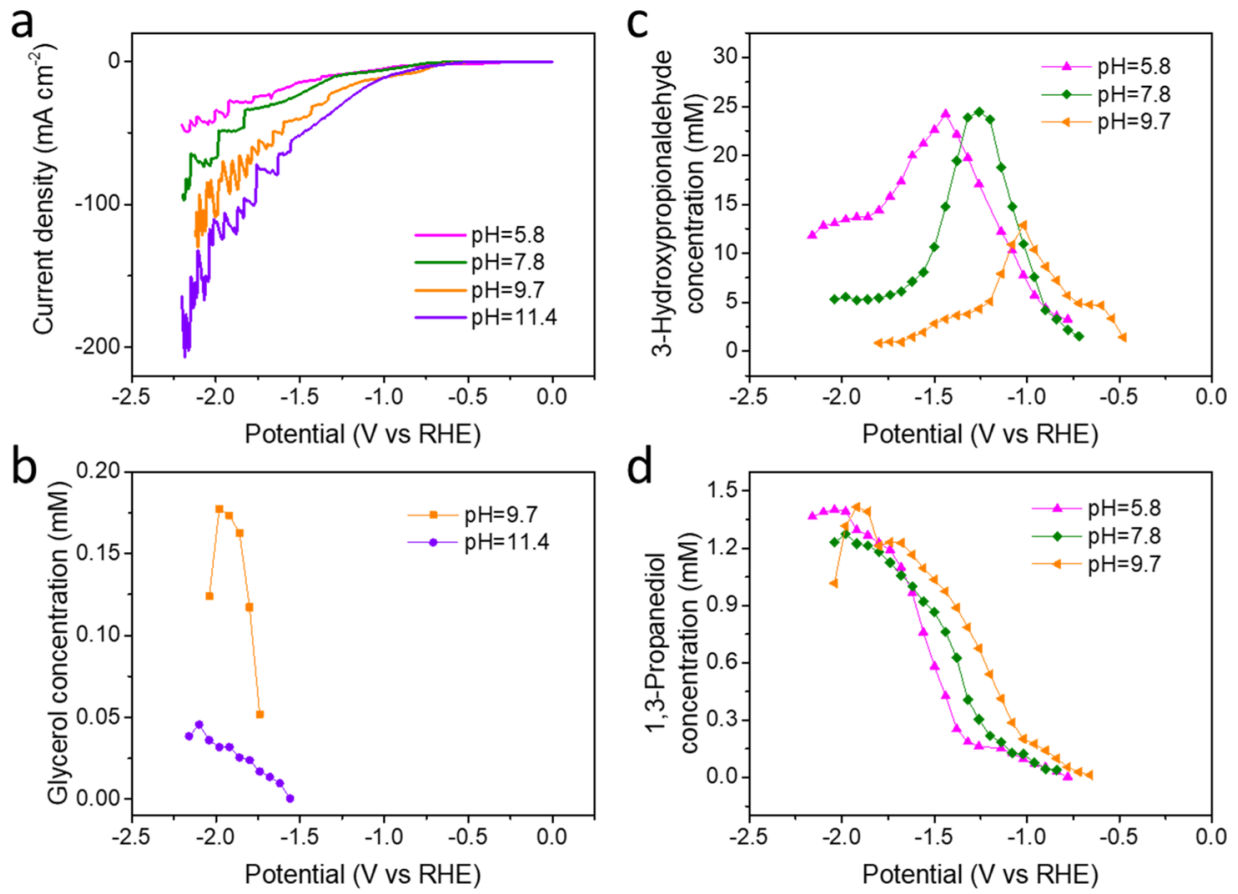

Figure 4. GA reduction in phosphate buffer electrolyte. (a) LSV at different $\mathrm{pH}$. The scan rate is $1.0 \mathrm{mV} \mathrm{s}^{-1}$. (b-d) Corresponding product concentrations of (b) glycerol, (c) 3-HPA, and (d) 1,3-PD as a function of the applied potential.

The reduction of GA in the buffer solution with $\mathrm{pH}$ range from 5 to 9 shows similar product distribution as that in the unbuffered electrolyte (Figure 4). We observed that the product concentration of 3-HPA and 1,3-PD shifted to positive potentials with increasing $\mathrm{pH}$ (Figure $4 \mathrm{c}, \mathrm{d}$ ). This trend is similar to what we observed for DHA reduction in buffer electrolytes. At a more alkaline condition, glycerol was additionally produced, while GA showed no activity in dehydroxylation reaction at $\mathrm{pH}=11.4$.

By comparing the results of DHA and GA reduction in both buffer and unbuffered electrolytes, we conclude that GA shows higher reduction activity than DHA. In the perchlorate electrolyte, the maximum concentration of the first product for GA reduction is about 10 times higher than that of DHA reduction.

In Situ FTIR Spectroscopy. As discussed above, we observed suppressed current during DHA/GA reduction at negative potentials, which we assign to a poisoning species formed on the electrode surface. To obtain more insight into the identity of the poisoning species, we performed in situ FTIR spectroscopy. The spectra were recorded with $50 \mathrm{mM}$ DHA (Figure 5) and $50 \mathrm{mM}$ GA (Figure 6) as a function of potential in $\mathrm{pH}=3$ electrolyte: $0.001 \mathrm{M} \mathrm{HClO}_{4}+0.099 \mathrm{M}$ $\mathrm{NaClO}_{4}$. The spectra of p-polarized light were collected to observe the adsorbed species.

For DHA reduction, we hold first potential at $0 \mathrm{~V}$ versus RHE to collect the reference spectrum. When we applied negative potentials from $0 \mathrm{~V}$ to $-1.1 \mathrm{~V}$ versus RHE, a positive band at $1736 \mathrm{~cm}^{-1}$ was observed, which could be assigned to $\mathrm{C}=\mathrm{O}$ stretching of the carbonyl group in $\mathrm{DHA},{ }^{21}$ indicating the consumption of DHA in the thin layer between the electrode and the prism. The peaks at 1431 and $1257 \mathrm{~cm}^{-1}$ are ascribed to $\mathrm{CH}_{3}$ and $\mathrm{CH}_{2}$ deformations and $\mathrm{C}-\mathrm{C}-\mathrm{H}$ deformation, respectively. ${ }^{22,23}$ These features can be attributed to either the reactant DHA or the acetol, that is the first reduction product (see transmission spectra of DHA and
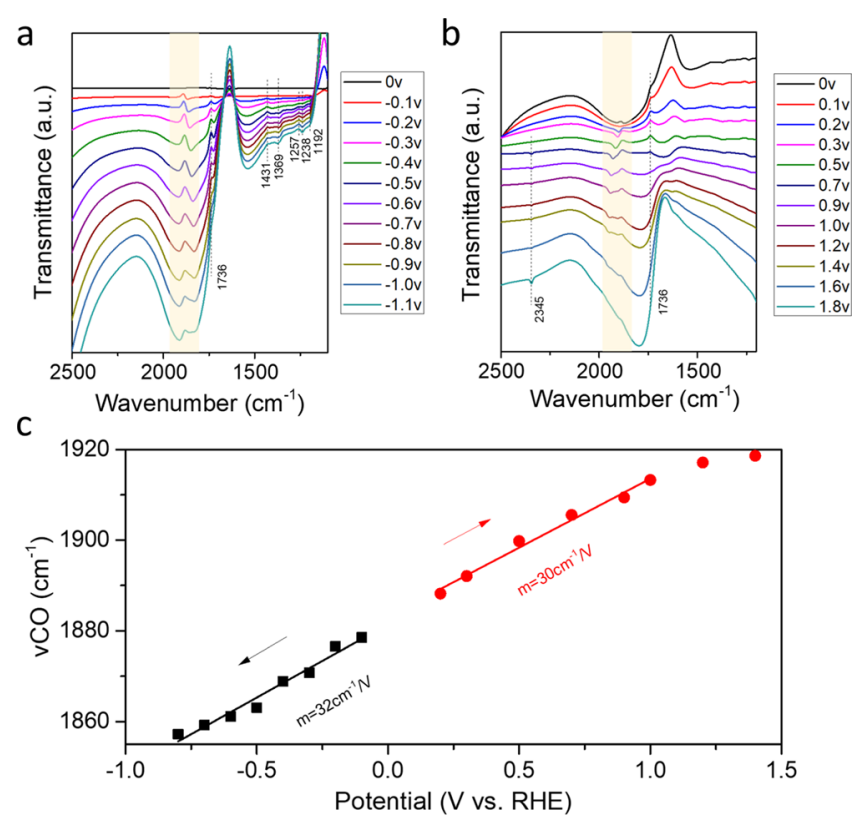

Figure 5. In situ FTIR spectra as a function of potential for $50 \mathrm{mM}$ DHA reduction on $\mathrm{Pd}$ electrode in $\mathrm{pH}=3: 0.001 \mathrm{M} \mathrm{HClO}_{4}+0.099$ $\mathrm{M} \mathrm{NaClO}$. Spectra were collected using p-polarized light. The reference spectrum was collected at $0 \mathrm{~V} v s$ RHE. (a) Spectra recorded from 0 to $-1.1 \mathrm{~V} v s$ RHE. (b) Spectra recorded from 0 to $1.8 \mathrm{~V} v s$ RHE directly after scanning to $-1.1 \mathrm{~V} v$ RHE as shown in (a). (c) Band position of the $\mathrm{CO}$ vibration as a function of the applied potential: the black squares correspond to the peak shift at negative potentials in (a); and the red circles correspond to the peak shift at positive potentials in $(\mathrm{b})$. The slope $(\mathrm{m})$ gives the Stark tuning of the adsorbate vibration.

acetol in Figure S9). The large band at around $1637 \mathrm{~cm}^{-1}$ is due to the $\mathrm{OH}$ bending from the water layer in the IR cell configuration. 

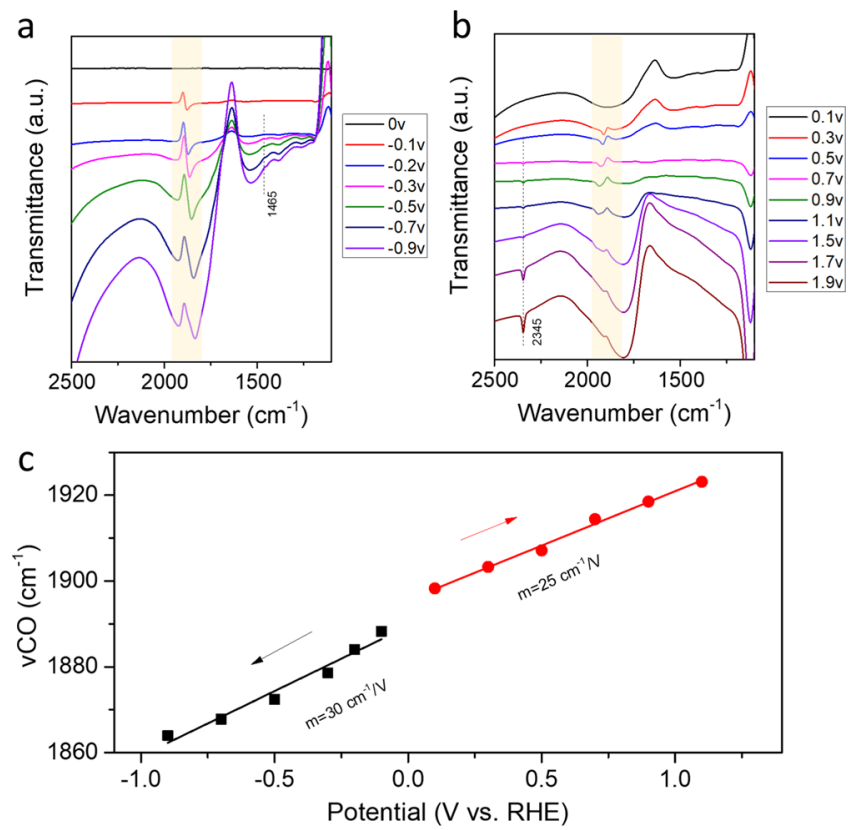

Figure 6. In situ FTIR spectra as a function of potential for $50 \mathrm{mM}$ GA reduction on Pd electrode in $\mathrm{pH}=3: 0.001 \mathrm{M} \mathrm{HClO}_{4}+0.099 \mathrm{M}$ $\mathrm{NaClO}_{4}$. Spectra were collected using p-polarized light. The reference spectrum was collected at $0 \mathrm{~V} v s$ RHE. (a) Spectra recorded from 0 to $-0.9 \mathrm{~V}$ vs RHE. (b) Spectra recorded from 0.1 to $1.9 \mathrm{~V}$ vs RHE directly after scanning to $-0.9 \mathrm{~V}$ vs RHE as shown in (a). (c) Band position of the $\mathrm{CO}$ vibration as a function of the applied potential: the black squares correspond to the peak shift at negative potentials in (a); and the red circles correspond to the peak shift at positive potentials in (b). The slope $(m)$ gives the Stark tuning of the adsorbate vibration.

The negative peak at $1369 \mathrm{~cm}^{-1}$ can be ascribed to the symmetric $\mathrm{CH}_{3}$ bending of acetol or acetone, ${ }^{23,24}$ the peak at $1238 \mathrm{~cm}^{-1}$ represents either the $\mathrm{C}-\mathrm{OH}$ bending of acetol or the asymmetric stretching of the carbon skeleton of acetone, and the peak at $1192 \mathrm{~cm}^{-1}$ corresponds to $\mathrm{C}-\mathrm{C}$ stretching of acetol. These features indicate the production of acetol and acetone, which is also confirmed by the transmission spectra (Figure S9). It is worth to note that the onset potential of the first product observed from the spectra is $c a$. $-0.4 \mathrm{~V}$ versus RHE, which is earlier than that in HPLC detection. This may be due to the higher sensitivity of FTIR technique or to the stronger $\mathrm{pH}$ gradients that exist near the electrode surface in the FTIR cell geometry.

In addition, we observed a conspicuous bimodal band at around $1880 \mathrm{~cm}^{-1}$ (Figure 5a). The peak shifts toward lower frequency with increasing negative potentials, presenting a Stark tuning slope of $32 \mathrm{~cm}^{-1} / \mathrm{V}$ (Figure $5 \mathrm{c}$ ). This species could be assigned to carbon monoxide $(\mathrm{CO})$ in a three-fold hollow surface site which is strongly adsorbed on the $\mathrm{Pd}$ surface. $^{25}$ This poisoning CO must stem from the dissociative adsorption of DHA.

It is worth to note that, as mentioned above, the local alkaline $\mathrm{pH}$ leads to side reactions, such as isomerization or aldol condensation. In order to exclude possible poisoning species due to these side-reaction products, we recorded transmission spectra of $50 \mathrm{mM}$ DHA in $0.1 \mathrm{M} \mathrm{NaOH}$ (Figure S10) for different standing times. No features were detected between 2000 and $1800 \mathrm{~cm}^{-1}$. We also mixed $50 \mathrm{mM} \mathrm{DHA}$ and $50 \mathrm{mM}$ GA in $0.1 \mathrm{M} \mathrm{NaOH}$, where the hexose is formed from trioses through aldol condensation reaction. ${ }^{26}$ However, there was no spectral peak at around $1880 \mathrm{~cm}^{-1}$ (Figure S11).

We then applied positive potentials to track the fate of the $\mathrm{CO}$ band (Figure $5 \mathrm{~b}$ ). The bimodal feature shifts to about $1890 \mathrm{~cm}^{-1}$ at $0.2 \mathrm{~V}$ versus RHE and presents a Stark tuning slope of $30 \mathrm{~cm}^{-1} / \mathrm{V}$ with increasing the positive potential (Figure 5c). At the potential of $0.7 \mathrm{~V}$ versus RHE, $\mathrm{CO}_{2}$ species with a band at $2345 \mathrm{~cm}^{-1}$ appeared. When the applied positive potentials are higher than $1.6 \mathrm{~V}$ versus $\mathrm{RHE}$, the $\mathrm{CO}$ feature gradually disappeared while $\mathrm{CO}_{2}$ intensity increased.

We concluded that the thin-layer configuration in this FTIR setup did not have any qualitative impact on the observation of adsorbed species for DHA reduction, as the FTIR results were consistent with what we observed from HPLC. Also, the spectra did not show major changes within the applied potential window.

GA reduction presents similar spectra to that of DHA reduction. We first held the potential at $0 \mathrm{~V}$ versus RHE to record the reference spectrum. With more negative potentials, no trace of $\mathrm{C}=\mathrm{O}$ stretching was detected at about $1730 \mathrm{~cm}^{-1}$ (Figure 6a), as was confirmed by the transmission spectra of GA (Figure S12). It indicates that GA exists as a cyclic dimer due to hemiacetal formation between the two molecules. ${ }^{27}$ The peak at $1465 \mathrm{~cm}^{-1}$ can be attributed to $\mathrm{OH}$ bending of the hemiacetal $\mathrm{OH}$ group. ${ }^{27}$

Likewise, we detected a bimodal feature at $1890 \mathrm{~cm}^{-1}$ with a Stark tuning slope of $30 \mathrm{~cm}^{-1} / \mathrm{V}$ (Figure 6c). We again attribute it to the triple-bonded $\mathrm{CO}$ adsorbed on the $\mathrm{Pd}$ electrode, which comes from the dissociation of GA or its dimer under applied potentials. We excluded the possible poisoning adsorbate to be generated from side reactions of GA in alkaline solution (Figure S13).

Figure $6 \mathrm{~b}$ shows the $\mathrm{CO}$ vibrational feature when the potential applied is from 0.1 to $1.9 \mathrm{~V}$ versus RHE. Analogous

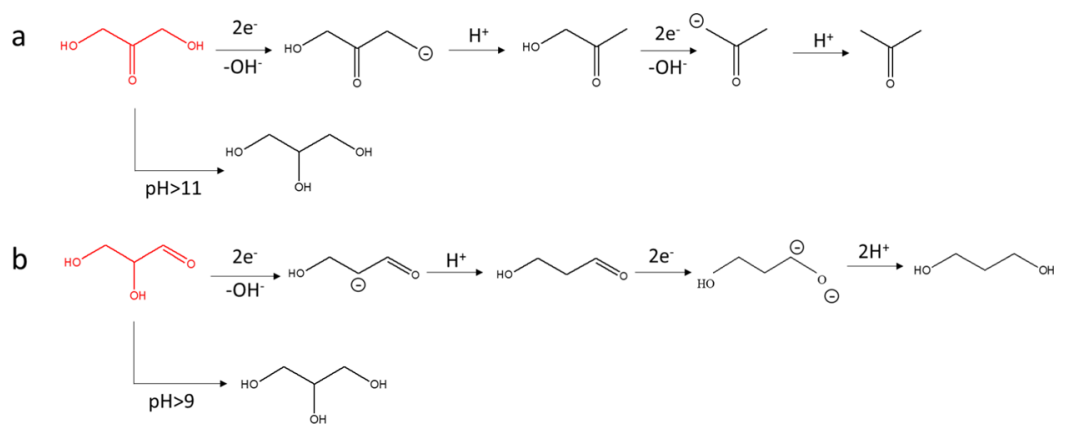

Figure 7. Proposed reaction pathways for (a) DHA and (b) GA reduction on a palladium electrode. 
to the observation for DHA, the CO feature presents a Stark tuning slope of $25 \mathrm{~cm}^{-1} / \mathrm{V}$. When the potential is more positive than $0.7 \mathrm{~V}$ versus $\mathrm{RHE}, \mathrm{CO}_{2}$ species appears because of the oxidation of the adsorbed $\mathrm{CO}$ and formate species in the thin layer.

To clarify the effect of $\mathrm{CO}$ poisoning species on DHA and GA reduction, we performed bulk electrolysis and showed the evolution of the reduction products with the reaction time (Figure S14). For DHA reduction, at $\mathrm{pH}=6$, the $\mathrm{FE}$ of acetol was decreased from $30 \%$ in the first reaction hour to $11 \%$ after a 3-hour reaction at $-0.9 \mathrm{~V}$ versus RHE. For GA reduction, at $\mathrm{pH}=6$, the final product 1,3-PD showed a remaining FE of $13 \%$ after a 3 -hour reaction at $-1.1 \mathrm{~V}$ versus RHE. The results indicate poisoning by adsorbed $\mathrm{CO}$ on the electrode surface.

Reaction Pathways. On the basis of the above results, we conclude that DHA reduction follows a two-step reaction pathway, as shown in Figure $7 \mathrm{a}$, where the $\mathrm{C}-\mathrm{OH}$ group, adjacent to the carbonyl group, is initially activated to form a negatively charged species, followed by proton transfer and dehydration to form acetol. We hypothesize the involvement of a negatively charged intermediate on the basis of the $\mathrm{pH}$ dependence observed. The further reduction of acetol follows the same dehydroxylation reaction to produce the final product, acetone.

GA reduction undergoes a similar dehydroxylation in the first step, where the electroreductive cleavage of $\mathrm{C}-\mathrm{OH}$ bond occurs, forming an anion intermediate (Figure $7 \mathrm{~b}$ ). It has also been suggested that such anion is generally in equilibrium with its enol form in the acid-base catalyzed reaction. ${ }^{12}$ In the acid electrolyte, the equilibrium shifts toward the nonreducible 3hydroxy-1-propenol; therefore, no further reduction occurs. This explains our result from online HPLC where at $\mathrm{pH}=1$, we only observed a negligible concentration of 3-HPA, while no $1,3-\mathrm{PD}$ was produced. On the other hand, at $\mathrm{pH}=3$ and 6 , the local alkaline $\mathrm{pH}$ near the electrode surface allows the acidbase equilibrium to shift toward the transformation into 3HPA, followed by further hydrogenation.

Finally, when the bulk $\mathrm{pH}$ is alkaline enough, the reduction of both DHA and GA can go through the alternative pathway that hydrogenates the carbonyl group directly to give glycerol as a final product.

\section{CONCLUSIONS}

The elucidation of a detailed mechanism of the reduction of saccharides is challenging due to the presence of many carbonyl and hydroxyl groups, leading to the involvement of multiple intermediates and reaction steps. In this work, we investigated the electrochemical reduction of the simplest monosaccharides, DHA and GA, under different $\mathrm{pH}$ conditions on a catalytic palladium electrode.

We found that both DHA and GA follow a two-step reduction process. For DHA reduction, the consecutive cleavage of $-\mathrm{OH}$ group in the $\alpha$-position of the carbonyl group gives the production of first acetol which is further reduced to acetone. It shows the lowest onset potential for both products at $\mathrm{pH}=1$. GA reduction follows a similar trend as DHA reduction, where the activation of $\mathrm{C}-\mathrm{OH}$ group adjacent to the carbonyl group gives 3-HPA as the first product. However, at $\mathrm{pH}=1$, the GA reduction activity is very low, and the equilibrium shifts toward the enol formation in acidic solution instead of the transformation to 3-HPA. Both DHA and GA reduction are $\mathrm{pH}$-dependent and generate more products under alkaline conditions. At more alkaline conditions $(\mathrm{pH}>11)$, another pathway toward direct hydrogenation of the carbonyl group occurs to produce glycerol.

From in situ FTIR spectra, we observed the formation of an adsorbed poisoning $\mathrm{CO}$ species which stems from dissociative adsorption of DHA/GA on the Pd electrode. Future research may tackle the problem of the poisoning species through electrode modification.

\section{ASSOCIATED CONTENT}

\section{Supporting Information}

The Supporting Information is available free of charge at https://pubs.acs.org/doi/10.1021/acscatal.0c04131.

Characterization of Pd electrode after the mechanical polishing; electrode effect on DHA electroreduction; chromatograms for DHA and GA reduction at $\mathrm{pH}=6$; reduction of acetol and 3-HPA on Pd electrode; bulk electrolysis for DHA and GA reduction; transmission spectra of DHA, acetol, acetone, GA, 3-HPA, and 1,3PD; yield and faradaic efficiency of DHA and GA reduction products; composition of the buffer electrolytes and their solution resistance; $\mathrm{pH}$ of the sample solution before and after reaction for DHA and GA reduction; and Faradaic efficiency of DHA and GA reduction products (PDF)

\section{AUTHOR INFORMATION}

\section{Corresponding Author}

Marc T. M. Koper - Leiden Institute of Chemistry, Leiden University, RA Leiden 2300, the Netherlands; 10 orcid.org/ 0000-0001-6777-4594; Email: m.koper@

chem.leidenuniv.nl

\section{Authors}

Zhiqin Liang - Leiden Institute of Chemistry, Leiden University, RA Leiden 2300, the Netherlands

Matias A. Villalba - Leiden Institute of Chemistry, Leiden University, RA Leiden 2300, the Netherlands

Giulia Marcandalli - Leiden Institute of Chemistry, Leiden University, RA Leiden 2300, the Netherlands

Kasinath Ojha - Leiden Institute of Chemistry, Leiden University, RA Leiden 2300, the Netherlands

Arthur J. Shih - Leiden Institute of Chemistry, Leiden University, RA Leiden 2300, the Netherlands

Complete contact information is available at:

https://pubs.acs.org/10.1021/acscatal.0c04131

\section{Notes}

The authors declare no competing financial interest.

\section{ACKNOWLEDGMENTS}

This research received funding from the Netherlands Organization for Scientific Research (NWO) in the framework of the fund New Chemical Innovations, project 731.015.204 ELECTROGAS, with financial support of Akzo Nobel Chemicals, Shell Global Solutions, Magneto Special Anodes (an Evoqua Brand) and Elson Technologies, and the Solar-toProducts program financed by The Netherlands Organization for Scientific Research (NWO) and by Shell Global Solutions International B.V. 


\section{REFERENCES}

(1) Nakagawa, Y.; Tomishige, K. Heterogeneous catalysis of the glycerol hydrogenolysis. Catal. Sci. Technol. 2011, 1, 179-190.

(2) Zhou, C.-H.; Beltramini, J. N.; Fan, Y.-X.; Lu, G. Q. Chemoselective catalytic conversion of glycerol as a biorenewable source to valuable commodity chemicals. Chem. Soc. Rev. 2008, 37, 527-549.

(3) Garcia, A. C.; Kolb, M. J.; van Nierop y Sanchez, C.; Vos, J.; Birdja, Y. Y.; Kwon, Y.; Tremiliosi-Filho, G.; Koper, M. T. M. Strong impact of platinum surface structure on primary and secondary alcohol oxidation during electro-oxidation of glycerol. ACS Catal. 2016, 6, 4491-4500.

(4) Garcia, A. C.; Birdja, Y. Y.; Tremiliosi-Filho, G.; Koper, M. T. M. Glycerol electro-oxidation on bismuth-modified platinum single crystals. J. Catal. 2017, 346, 117-124.

(5) Kwon, Y.; Birdja, Y.; Spanos, I.; Rodriguez, P.; Koper, M. T. M. Highly selective electro-oxidation of glycerol to dihydroxyacetone on platinum in the presence of bismuth. ACS Catal. 2012, 2, 759-764.

(6) Bard, A.; Stratmann, M.; Schäfer, H. Encyclopedia of Electrochemistry, Organic Electrochemistry; Wiley-VCH: Weinheim, Germany, 2004; Vol. 8.

(7) Sauter, W.; Bergmann, O. L.; Schröder, U. Hydroxyacetone: A Glycerol-Based Platform for Electrocatalytic Hydrogenation and Hydrodeoxygenation Processes. ChemSusChem 2017, 10, 3105-3110.

(8) James, O. O.; Sauter, W.; Schröder, U. Towards selective electrochemical conversion of glycerol to 1, 3-propanediol. RSC Adv. 2018, 8, 10818-10827.

(9) Hunsom, M.; Saila, P. Product distribution of electrochemical conversion of glycerol via Pt electrode: Effect of initial $\mathrm{pH}$. Int. J. Electrochem. Sci. 2013, 8, 11288-11300.

(10) Lee, C. S.; Aroua, M. K.; Daud, W. M. A. W.; Cognet, P.; PeresLucchese, Y.; Ajeel, M. A. Selective electroreduction of glycerol to 1, 2-propanediol on a mixed carbon-black activated carbon electrode and a mixed carbon black-diamond electrode. Bioresources 2018, 13, $115-130$.

(11) Fedoroňko, M.; Königstein, J.; Linek, K. Polarographic and preparative electroreduction of DL-glyceraldehyde and dihydroxyacetone. Collect. Czech. Chem. Commun. 1965, 30, 4297-4306.

(12) Fedoroňko, M. Electroreduction of DL-glyceraldehyde and Dforms of its 2, 3-di-O-methyl-propylidene and 2, 3-O-isopropylidene derivatives. Chem. Pap. 1987, 41, 767-776.

(13) Correia, A. N.; Mascaro, L. H.; Machado, S. A. S.; Avaca, L. A. Active surface area determination of Pd-Si alloys by H-adsorption. Electrochim. Acta 1997, 42, 493-495.

(14) Fang, L.-l.; Tao, Q.; Li, M.-f.; Liao, L.-w.; Chen, D.; Chen, Y.-x. Determination of the real surface area of palladium electrode. Chin. J. Chem. Phys. 2010, 23, 543-548.

(15) Kwon, Y.; Koper, M. T. M. Combining Voltammetry with HPLC: Application to Electro-Oxidation of Glycerol. Anal. Chem. 2010, 82, 5420-5424.

(16) Chen, Q.-A.; Ye, Z.-S.; Duan, Y.; Zhou, Y.-G. Homogeneous palladium-catalyzed asymmetric hydrogenation. Chem. Soc. Rev. 2013, 42, 497-511.

(17) Kwon, Y.; Koper, M. T. M. Electrocatalytic hydrogenation and deoxygenation of glucose on solid metal electrodes. ChemSusChem 2013, 6, 455-462.

(18) De Wit, G.; Kieboom, A. P. G.; Van Bekkum, H. Enolisation and isomerisation of monosaccharides in aqueous, alkaline solution. Carbohydr. Res. 1979, 74, 157-175.

(19) Berl, W. G.; Feazel, C. E. The Kinetics of Hexose Formation from Trioses in Alkaline Solution1. J. Am. Chem. Soc. 1951, 73, 20542057.

(20) Schouten, K. J. P.; Kwon, Y.; Van der Ham, C. J. M.; Qin, Z.; Koper, M. T. M. A new mechanism for the selectivity to $C_{1}$ and $C_{2}$ species in the electrochemical reduction of carbon dioxide on copper electrodes. Chem. Sci. 2011, 2, 1902-1909.

(21) Chen, S.-H.; Hiramatsu, H. Tautomer Structures in KetoseAldose Transformation of 1, 3-Dihydroxyacetone Studied by Infrared
Electroabsorption Spectroscopy. J. Phys. Chem. B 2019, 123, 1066310671 .

(22) Nickless, E. M.; Holroyd, S. E.; Hamilton, G.; Gordon, K. C.; Wargent, J. J. Analytical method development using FTIR-ATR and FT-Raman spectroscopy to assay fructose, sucrose, glucose and dihydroxyacetone, in Leptospermum scoparium nectar. Vib. Spectrosc. 2016, 84, 38-43.

(23) Harty-Major, S. Investigation of the Chemistry of 1hydroxyacetone by Fourier Transform Infrared Spectroscopy. McGill University, 1999.

(24) Dellepiane, G.; Overend, J. Vibrational spectra and assignment of acetone, $\alpha \alpha \alpha$ acetone- $\mathrm{d}_{3}$ and acetone- $\mathrm{d}_{6}$. Spectrochim. Acta 1966, 22, 593-614.

(25) Solomun, T. Coverage Dependence of CO Electrosorption on Palladium: An in-situ IR Study. Ber. Bunsen-Ges. 1986, 90, 556-558.

(26) Fischer, H. O. L.; Baer, E. Synthese von d-Fructose und dSorbose aus d-Glycerinaldehyd, bzw. aus d-Glycerinaldehyd und Dioxy-aceton; über Aceton-glycerinaldehyd III. Helv. Chim. Acta 1936, 19, 519-532.

(27) Kobayashi, Y.; Igarashi, T.; Takahashi, H.; Higasi, K. Infrared and raman studies of the dimeric structures of 1, 3-dihydroxyacetone, D(+)-and DL-glyceraldehyde. J. Mol. Struct. 1976, 35, 85-99. 